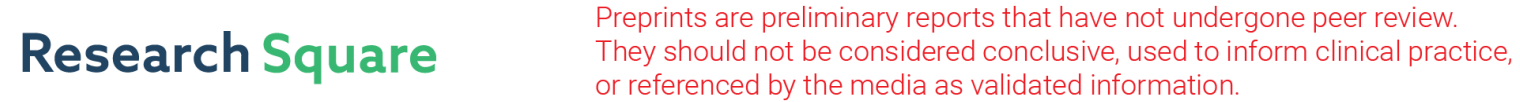 \\ Viral Strategies for Targeting Spinal Neuronal Subtypes in Adult Wild-Type Rodents \\ Jaspreet Kaur \\ University of Copenhagen \\ Rune W. Berg ( $\nabla$ runeb@sund.ku.dk) \\ University of Copenhagen
}

\section{Research Article}

Keywords: spinal, interneurons, AAV, GABA

Posted Date: November 30th, 2021

DOI: https://doi.org/10.21203/rs.3.rs-1050494/v1

License: (c) (i) This work is licensed under a Creative Commons Attribution 4.0 International License. Read Full License 


\title{
Viral strategies for targeting spinal neuronal subtypes in adult wild-type rodents
}

\author{
Jaspreet Kaur, ${ }^{1}$ and Rune W. Berg ${ }^{1 *}$ \\ ${ }^{1}$ Department of Neuroscience, Faculty of Health and Medical Sciences, University of Copenhagen \\ Blegdamsvej 3, 2200 Copenhagen, Denmark \\ *Correspondence; E-mail: runeb@sund.ku.dk (RWB)
}

\begin{abstract}
Targeting specific subtypes of interneurons in the spinal cord is primarily restricted to a small group of genetic model animals. Since the development of new transgenic model animals can be expensive and labor intensive, it is often difficult to generalize these findings and verify them in other model organisms, such as the rat, ferret or monkey, that may be more beneficial in certain experimental investigations. Nevertheless, endogenous enhancers and promoters delivered using an adeno-associated virus (AAV) have been successful in providing expression in specific subtypes of neurons in the forebrain of wildtype animals, and therefore may introduce a shortcut. GABAergic interneurons, for instance, have successfully been targeted using the $m D / x$ promotor, which has recently been developed and is now widely used in wild type animals. Here, we test the specificity and efficiency of the $\mathrm{mDlx}$ enhancer for robust targeting of inhibitory interneurons in the lumbar spinal cord of wild-type rats. Since this has rarely been done in the spinal cord, we also test the expression and specificity of the CamKIla and hSynapsin promoters. We found that $m D / x$ does in fact target many neurons that contain an enzyme for catalyzing GABA, the GAD-65, with high specificity and a small fraction of neurons containing an isoform, GAD-67. Expression was also seen in some motor neurons. Viral injections using the CamKIla enhancer infected in some glutamatergic neurons but also GABAergic neurons, whereas hSynapsin targets almost all the neurons in the lumbar spinal cord.
\end{abstract}

Genetic tools have been instrumental for teasing apart circuits in the central nervous system ${ }^{1}$. Targeting specific subpopulations of neurons have helped addressing questions whether the functional organization of the nervous system can be linked to the genetic identity of neurons. Such investigations are often accomplished using transgenic animals, which are expensive to acquire, labor intensive to breed and often restricted to only a single model organism, which is most often the mouse. As a consequence, it is difficult to assess the translational potential of these findings in other model organisms that are less mainstream albeit closer to humans, such as the rat, ferret or monkey. Therefore genetic tools that can be delivered via virus to target specific cellular subtypes using an endogenous promotor or enhancer without the need of transgenic animals would not only be helpful, but also scientifically important by ensuring diversity in model organisms. Cell-type genetic drivers that are based on endogenous gene expression could also be used in combination with the transgenic tools to increase the options of manipulations, e.g. to combine optogenetics and calcium imaging, or to image two populations simultaneously.

Generic endogenous promotors like $\mathrm{Ca}^{2^{+}} /$calmodulin-dependent protein kinase II $\alpha$ (CamKIIa), human synapsin 1 gene $(\text { HSyn } 1)^{2}$ and the Thy 1 gene have been applied extensively for targeting mature neurons of wild-type animals. Nevertheless, these promotors target either primarily excitatory neurons or all neurons non-specifically, and alternative promotors are in demand. The novel transcriptional enhancer mDlx is based on the distal-less (Dlx) homeobox gene group, which is a key regulator for inhibitory interneurons ${ }^{3}$. The Dlx genes are important in both invertebrates and vertebrates ${ }^{4}$ and are involved in expression of interneurons in the telencephalon, that primarily use Gamma-amino-byturic acid (GABA) as neurotransmitter ${ }^{5}$. The Dlx genes are necessary for expression of the enzyme to catalyze GABA (GAD65 and 67) and general GABAergic synaptogenesis and dendritogenesis ${ }^{3,5}$. The $\mathrm{mDlx}$ enhancer has been demonstrated useful in investigations of e.g. the ferret visual cortex ${ }^{6}$, but it is unknown whether the specificity is found in the spinal cord ${ }^{7}$. There is a large heterogeneity in spinal interneurons $s^{8,9}$, for which the functional role is unclear. Inhibitory interneurons in the spinal cord has been proposed to be providing a counter-balance of excitation ${ }^{10-12}$ yet their role also remain unclear. The Dlx homeobox gene group is known to mark the neural crest, the telencephalic structures and placodes and primarily expressed in telencephalic structures ${ }^{13}$, hence it is unsure whether this enhancer will be specific in the spinal cord.

Here, we tested the mDlx enhancer for marking inhibitory interneurons in the spinal cord, using an adeno-associated virus, which was injected in the lumbar spinal cord of wild-type rats. Furthermore, we tested the promotors hSynapsin1 and CaMKIIa. The latter is known to target primarily excitatory neurons in the forebrain ${ }^{14,15}$, but the expression characteristics in the spinal cord are less well-established. First we verified the expression of AAVs carrying these promotors and then quantify 


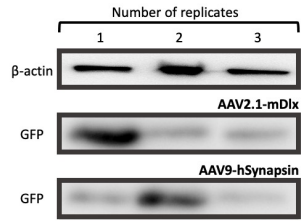

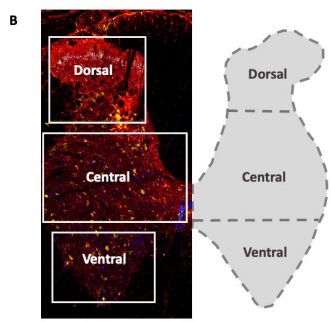

Fig. 1. Expression of AAV viruses in the lumbar spinal cord of wild-type Wistar adult rats. (A) Western blot images showing expression of AAV2.1-mDlx-GCaMP6f-Fishell-2 (N=3) and AAV9-hSynapsin-soCoChR-GFP (N=3) where N represents the number of rats/replicates used and beta-actin was a house-keeping protein. Full length western blots are shown in the supplementary Fig. 1 (A and B) where the cropped sections (shown here in A) are highlighted by black boxes. Expression of the third virus, AAV9-CamKIIa-ChrimsonR-mScarlet-Kv2.1, was very strong and therefore confirmation of viral expression was directly performed by visualising the spinal cord sections under the fluorescent microscope (image not shown). Note that an example of fluorescent virus expression in comparison to control spinal cord with no virus expression has shown in supplemental Fig. 1 (C). (B) Sample image of immunohistochemistry with a partitioning in dorsal (D, 560X720 $\mathrm{m}^{2}$ ), central $\left(\mathrm{C}, 590 \mathrm{X} 1070 \mu \mathrm{m}^{2}\right)$ and ventral $\left(\mathrm{V}, 460 \mathrm{X} 770 \mu \mathrm{m}^{2}\right)$ part of the lumbar spinal cord.

the expression in the dorso-ventral axis (Fig. 1). The expression specificity was evaluated using immunohistochemistry.

\section{Results}

An initial verification of viral expression in the spinal cord was performed using western blots, which showed a substantial expression of all the AAV viruses used in the study (Fig. 1 and Supplementary Fig. 1). Subsequently, the expression was reconfirmed by slicing the fixed spinal cords and visualized under the fluorescent microscope before performing immunostaining. Immunohistochemistry was performed on the slices which showed fluorescent signal from the AAV viral expression.

\section{mDIx expression primarily in GAD65 neurons}

We tested whether the $\mathrm{mDlx}$ enhancer that has been developed to target GABAergic interneurons in the forebrain ${ }^{7}$ can also target GABAergic neurons in the lumbar spinal cord of adult rats. The virus carried a fluorescent reporter, the green fluorescent protein (GFP). However, before testing the virus in the rat spinal cord we re-checked its expression in the mice brain (Supplemental Fig. 2) where the virus showed significant expression. To further inspect the GABAergic identity of virus expressed cells in mice prefrontal cortex we used a biomarker to label the PAX2 transcription factor, which has been associated with inhibitory interneurons ${ }^{16}$. PAX2 showed colocalization with GFP labelled neurons (Supplemental Fig. 2) whereas no colocalization with GAD67 biomarker.

Later we tested the mDlx promoter in rat lumbar spinal cord. The expression of GFP was compared with GABAergic neurons using anti-bodies for both isoforms of the enzyme glutamate decarboxylase 65 (GAD65) and 67 (GAD67) as GABAergic biomarkers (Fig. 2). We found that majority of GFP+ neurons colocalized with GAD65+ neurons, as indicated by punctuate structures in the cell bodies. There was approximately $80 \%$ of colocalization of GFP+ neurons and GAD65+ in the dorsal (D), central (C) and ventral (V) regions (Fig. 2 A-C). In constrast, neurons expressing the isoform, GAD67+, had only 5-10\% of co-localization with the GFP+ neurons (Fig. 2D and E). GAD67 were present either as punctuate structures or in the somata. Neurons with clear staining for GAD67 were abundantly present in the lamina 2, and virtually none of these had expression of GFP (Fig. 2D). To further investigate the colocalization between GFP and GABAergic neurons, we analysed the data using a more quantitative measure of protein colocalization where values of the original images (top row, Fig. 3A) above a certain threshold in one image was multiplied by the similar values in the other image (bottom row, fig. 3A). This provides an objective measure of the correlation of the location of two fluorescent proteins ${ }^{17}$, whose value depends on the choice of threshold (Fig. 3B-C). Regardless of the choice, the values were quite high (Fig. 3B). To further inspect the cell type, we used PAX2 and found that approximately 15\%-25\% of PAX2+ neurons in the D, C and V regions colocalize with GFP+ neurons (Supplementary Fig. $3)$.

We also observed some ventrally located GFP+ neurons that appeared to be motor neurons. To investigate this further, we performed a ChAT-immunolabeling (not shown) as well as an immunolabelling of neurofilament (SMI32), since it is known that motor neurons have neurofilaments in abundance (Supplementary Fig. 4). The ventrally located GFP+ neurons had large 

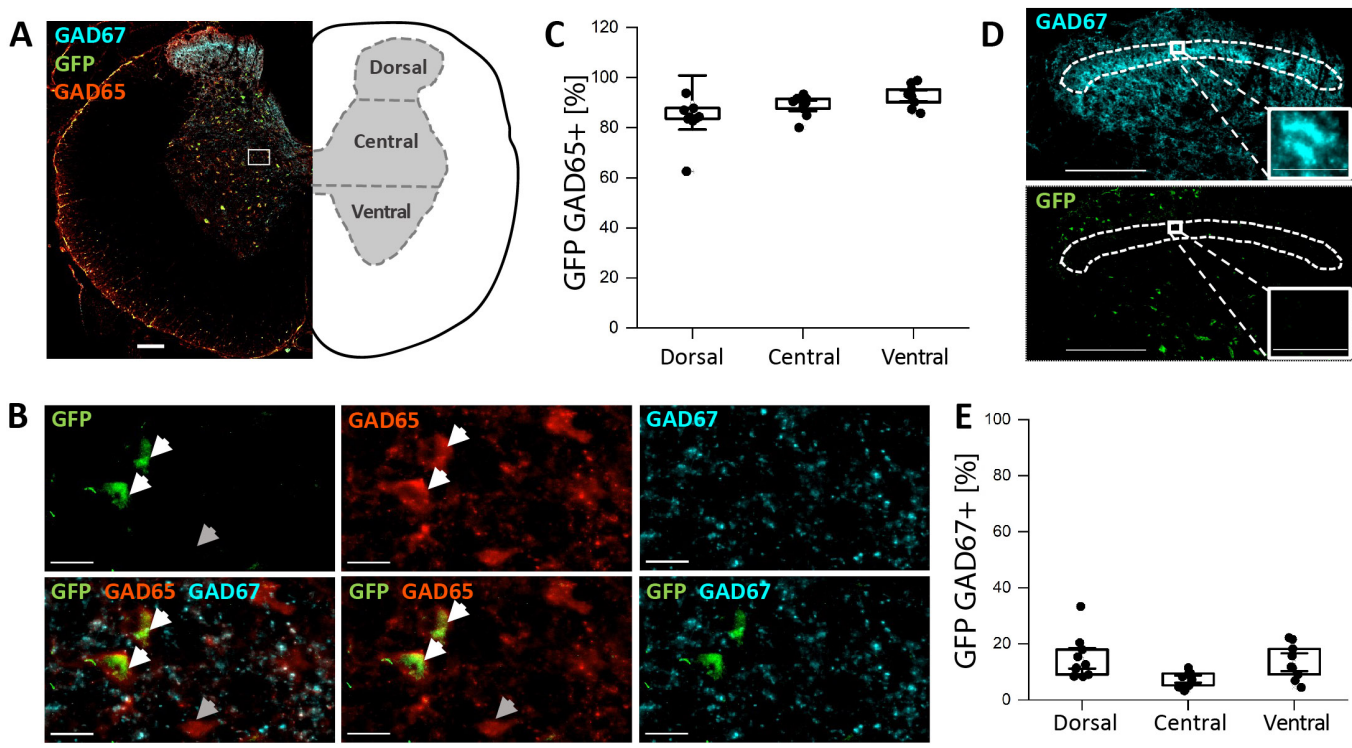

Fig. 2. The mDlx enhancer primarily target GAD65-expressing neurons in the lumbar spinal cord. (A) Sample tissue section and illustration of spinal cord. The mDlx expression is associated with a green fluorescent protein (GFP) as a reporter. The GAD65 (red) and GAD67 (cyan) are stained by immunolabelling. (B) Highlighted section in the central region in panel A (shown as zoomed-in images in panel B) indicates colocalization between GFP and GAD65, but not GAD67. (C) Fraction of colocalization between GFP-positive cells and cells containing GAD65 in the three dorsoventral regions $(\mathrm{N}=3, \mathrm{n}=3$; where $\mathrm{N}$ is the number of spinal cords used and $\mathrm{n}$ designates the number of sections analysed per spinal cord). (D) The dense layer of GAD67-containing neurons in the dorsal horn has little or no colocalization with GFP. (E) Cell count (shown as \%) of the colocalization in the three regions between GAD67 and GFP positive cells $(\mathrm{N}=3, \mathrm{n}=3)$.

somata and were most likely motor neurons. Hence, we conclude that the neurons that express GFP with mDlx as enhancer also express the virus in motor neurons.

\section{CamKIla promotor targets some excitatory neurons in the spinal cord}

Besides investigating the expression of the mDlx enhancer, we also inspected the AAV expression using the CaMKIIa enhancer. We used a different fluorescent reporter for the expression of AAV virus with CaMKIIa promotor, which is a strong red fluorescence protein (mScarlet). To determine the fraction of neurons that had expression of $\mathrm{mScarlet}$, we labeled the neurons using NeuN biomarker and counted the fraction that colocalized in D, C, and V regions (Fig. 4A and B). We identified roughly $5 \% \mathrm{mScarlet}+$ neurons in the D, $25 \%$ in the central and $40 \%$ in the ventral region (Fig. 4B). Next, we tested whether the $\mathrm{mScarlet+}$ neurons had a potential overlap with proteins that are distinctly associated with glutamatergic neurons. First, we tested for overlap with the vesicular glutamate transporters 1 and 2 (VGluT1 and VGluT2) (Fig. 4C and D). However, these transporters are primarily marking the axons and synaptic terminals ${ }^{18}$, which make it difficult to identify the type of cell. Anti-VGluT1 seemed to have occasional staining with in soma (top left, Fig. 4C) but the VGluT2 was largely indicated on dendrites outside the soma (bottom right, Fig. 4C). Counting the mScarlet+ cells that receive synaptic contacts we found $50-60 \%$ of these receiving inputs from VGluT1 and almost all the mScarlet+ neurons receive inputs from VGluT2. VGluT2 is abundantly present in the gray matter, hence resulting in a nebulous staining in the space surrounding the somata (Fig. 4C). The colocalization was further quantified in the same manner as above (Fig. 3). The colocalization using the threshold correlation was low regardless of where the threshold was set (Fig. 5). This was, however, expected even if the mScarlet positive cells were VGluT2 positive since there is a poor expression of mScarlet in the synaptic terminals.

Another indicator of glutamatergic input to neurons is antibodies for the AMPA receptors, GluA1 and GluA2 (Supplementary Fig. 5). We observed that around 20-30\% of mScarlet+ neurons contain GluA1 (Supplementary Fig. 5A-C) at the intracellular sites however it was challenging to trace the co-expression GluA2 with RFP+ neurons as GluA2 was also excessively present (Supplementary Fig. 5B bottom right image) in the spinal cord. These data demonstrate that the given AAV virus with CaMKIIa promotor receives glutamatergic inputs and GluA1/2 expression at various intracellular sites.

To inspect whether some of the CamKIIa-driven infection was occurring in inhibitory neurons, the infection was compared with immunostaining of GAD65. There was some overlap, but not as strong as with the mDlx-driven neurons (compare Fig. 3 and 6). 

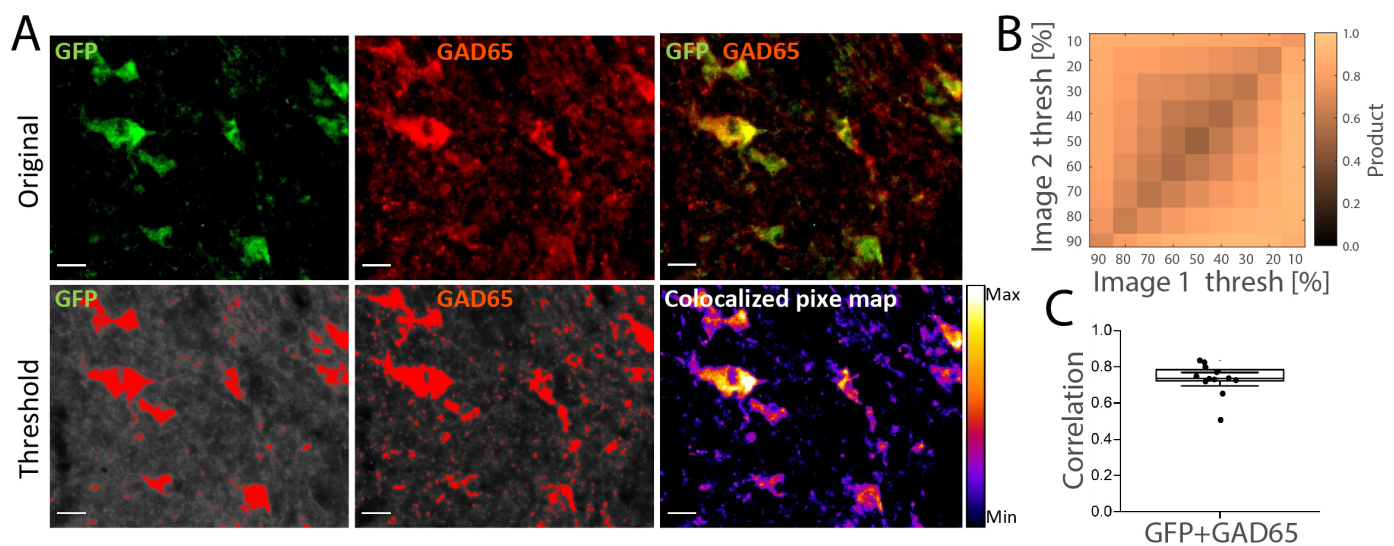

Fig. 3. mDlx enhancer has expression in GABAergic neurons, specifically the GAD65+ neurons. (A) Top row: $\mathrm{mDlx}$ reporter (GFP, green) and the GAD65 (red) staining has colocalization, Bottom row: thresholded versions of top row, with the overlap in heat map (right) (scale bar $=20 \mu \mathrm{m}$ ). (B) Varying the threshold and calculating the colocalization as correlation (C). Note $\mathrm{N}=4, \mathrm{n}=3$ where $\mathrm{N}$ designates the number of spinal cords used and $\mathrm{n}$ indicates the number of slices analysed per spinal cord.

\section{Targeting neurons using hSynapsin1 promotor}

We also tested the expression using the hSyn1 promotor in the spinal cord with expression of GFP and immunolabelled with NeuN. We observed that amongst total NeuN+ neurons nearly $45 \%$ of the GFP+ neurons in the D and approximately $90 \%$ in the $\mathrm{C}$ and $\mathrm{V}$ were showing co-expression with $\mathrm{NeuN}$ (Fig. 7). The extent of the colocalization was further quantified similar to the above using the threshold of the fluorescent signal (Fig. 8) and with similar strong correlation. These data indicate most of the spinal cord neurons could be targeted using hSyn 1 .

\section{Discussion}

Spinal interneurons are very diverse and there are many subtypes of GABAergic interneurons involved in e.g. the motor circuitry $^{8,9,19}$. For instance, the V1 inhibitory interneuron is genetically diverse with more than 50 subpopulations ${ }^{20}$ and tools investigating their function is in demand, especially for verification of the translational potential of the experiments done in transgenic model species. Here, we test three enhancers for specificity in well-defined subtypes of neuron. The plasmids were delivered using adeno-associated vira injected into the lumbar enlargement of rats. We found that the mDlx enhancer does not induce detectable expression in GABAergic neurons in the superficial layers that are known for high content of both GAD65 and GAD67 $7^{21,22}$. In the central and ventral layers, where there are less dense labeling there was a considerable colocalization of somata that had strong labeling of GAD65 rather than GAD67 and the reporter protein for mDlx (GFP). GABAergic neurons in central regions have previously been identified and suggested involved in parasympathic inhibition ${ }^{23}$. Nevertheless, there was also some expression in motoneurons using the mDlx enhancer whereas hSynapsin 1 promotor seems to target most of the spinal neurons. The CamKIIa promotor expresses in some GluA1 and VGluT1 neurons whereas most of the neurons are receiving VGluT2 inputs. To properly identify the infected cells, future studies with in situ hybridization to detect mRNA for VGluT2 $2^{24}$ are crucial.

\section{Materials and Methods}

\section{Animals and ethical statement}

The study is reported in accordance with ARRIVE guidelines. All experiments were approved by the Danish veterinary and food administration (animal research permission number 2019-15-0201-00018) and according to guidelines of the Council of the European Union (86/609/EEC).

Wild-type adult Wistar rats obtained from Charles River Laboratories were used for the experiments. Animals were housed in pairs at the animal facility and maintained in 12 light/12 dark cycle. Male rats were used however sex was not a crucial element of the study. Animals were habituated for at least 10 days before starting the experiment.

\section{Viral constructs}

To explore an AAV viral technique and to target specific neuronal types in lumbar spinal cord of wild-type rats we used three different types of AAV viruses: AAV2.1-mDlx-GCaMP6f-Fishell-2, AAV9-CamKIIa-ChrimsonR-mScarlet-Kv2.1 and 
A
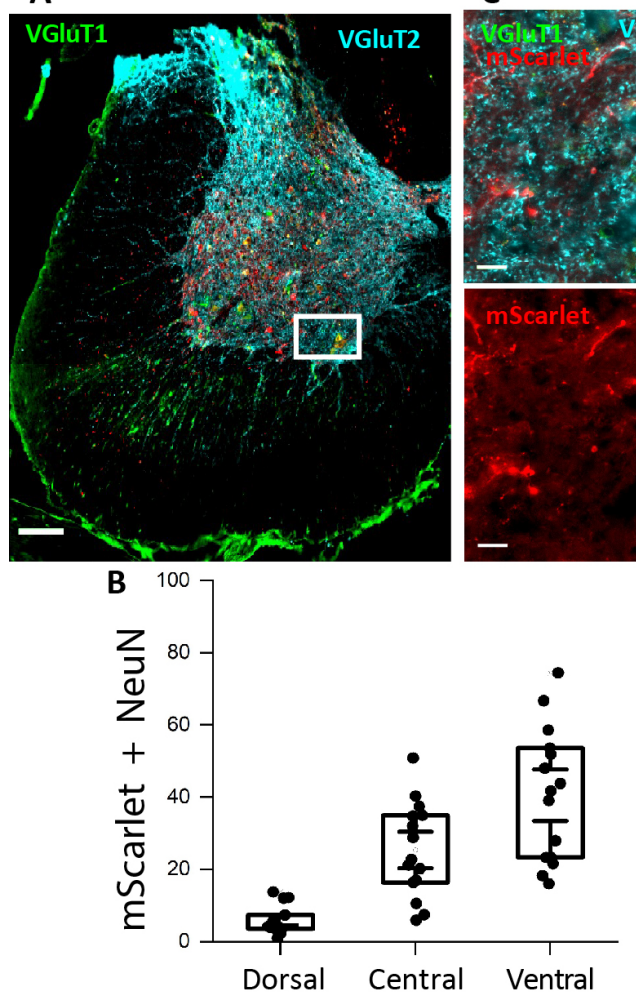

C
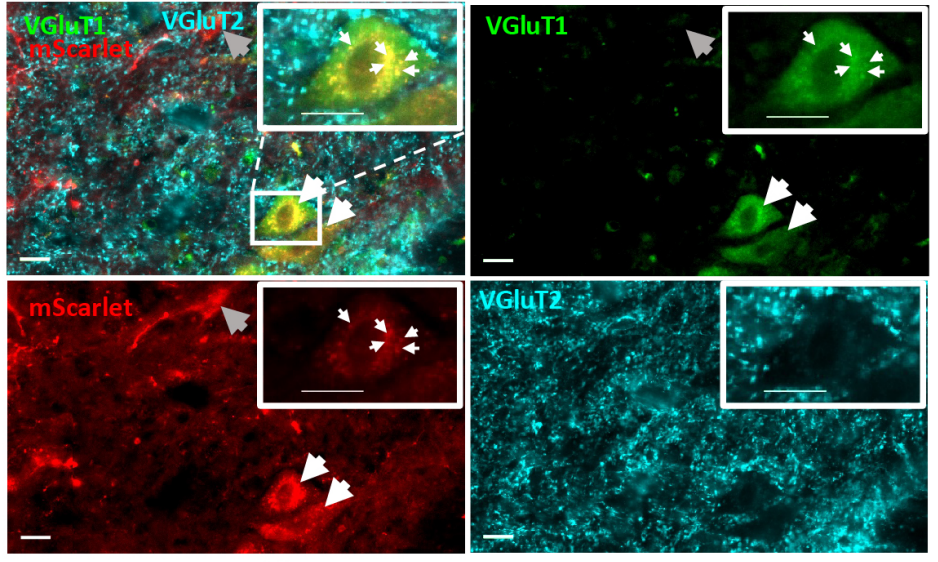

D

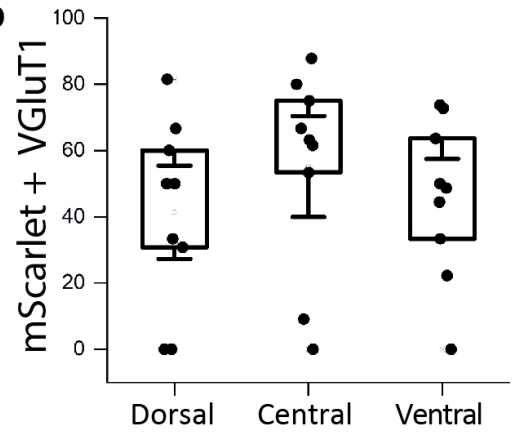

Fig. 4. Infection of spinal neurons with CamKIIa promoter primarily in the central and ventral regions. (A) Overview of infection (red, mScarlet) combined with the immunostaining of VGlut1 and 2. (B) Counting neurons that colocalized with the cells expressing $\mathrm{mScarlet}(\mathrm{N}=6, \mathrm{n}=3)$. (C) Zoomed in image of highlighted region in $(\mathrm{A})$ and the immunostaining of VGlut1 and 2 (green and cyan), indicates some overlap with VGlut1, whereas the VGlut2 primarily stains the synaptic terminals. (D) Box plot of the colocalized cell count with VGlut1 positive neurons $(\mathrm{N}=3, \mathrm{n}=3)$. Scale bar $=200 \mu \mathrm{m}$ in image A and 20 $\mu \mathrm{m}$ in the insets. Note that $\mathrm{N}$ is the number of spinal cords and $\mathrm{n}$ indicates the number of slices analysed per spinal cord.

AAV9-hSynapsin-soCoChR-GFP. The AAV2.1-mDlx-GCaMP6f-Fishell-2 was produced by incorporating the plasmid pAAVmDlx-GCaMP6f-Fishell-2, which is commercially available (Addgene.org) ${ }^{7}$ into a adeno-associated virus of serotype 2 . The pAAV-mDlx-GCaMP6f-Fishell-2 was a gift from Gordon Fishell (Addgene plasmid 83899; http://n2t . net/addgene: 83899; RRID : Addgene_83899). The pAAV-CamKIIa-ChrimsonR-mScarlet-KV2.1 was a gift from Christopher Harvey (Addgene plasmid 124651; http://n2t.net/addgene:124651;RRID:Addgene_124651), which was incorporated into a AAV of serotype 9. The pAAV-hSynapsin-soCoChR-GFP was a gift from Edward Boyden (Addgene plasmid 107708 ; http://n2t.net/addgene:107708;RRID:Addgene_107708), which was incorporated into an AAV of serotype 9 . In all injections the titer of the virus was $1 \cdot 10^{13}$ infectious units per $\mathrm{mL}$.

\section{Laminectomy and AAV virus injection}

Rats were anesthetized under isofluorane (1-3\% in oxygen) and placed in the stereotax frame on a heating pad with temperature controller. Laminectomy was performed at the thoraco-lumbar (T12-L1) part of the vertebrae, although similar alternative procedure has recently been suggested in mice ${ }^{25}$. The animals received an injection of AAV virus (1500-2000 nl) dorso-ventrally and rostro-caudally at the rate of $15 \mathrm{nl} / \mathrm{min}$ using a sharp glass capillary (inner diameter $=0.53 \mathrm{~mm}$ and outer diameter $=1.14$ $\mathrm{mm}$, Neurostar GmbH, Tubingen, Germany), which was left in place for 3-5 min after the injection to prevent the backflow ${ }^{26}$. Laminectomy site was covered, skin was sutured and the rats were housed in their cages for recovery. Post-operative care was provided via buprenorphine mixed with nutella (sublingual tablets $0.2 \mathrm{mg}$ crushed to powder and mixed with $1 \mathrm{~g}$ nutella, Dose $=0.4 \mathrm{mg} / \mathrm{kg}$, every $12 \mathrm{~h}$ for 3 days) and carprofen (subcutaneously (s.c.), $5 \mathrm{mg} / \mathrm{kg}$, once a day for 5 days) as analgesic and anti-inflammatory and baytril (s.c., $5 \mathrm{mg} / \mathrm{kg}$, once a day for 10 days) as antibiotic drugs. Animals used in the study showed no signs of distress and were monitored twice a day for 3 consecutive days and later once a day for 7 days.

For further confirmation of mDlx expression, fixed brains of C57BL/6N mice with mDlx injection in the prefrontal cortex were provided (as gift) by Dr. David Paul Drucker Woldbye and Amaia de Diego Ajenjo. Brains were sectioned and sections 

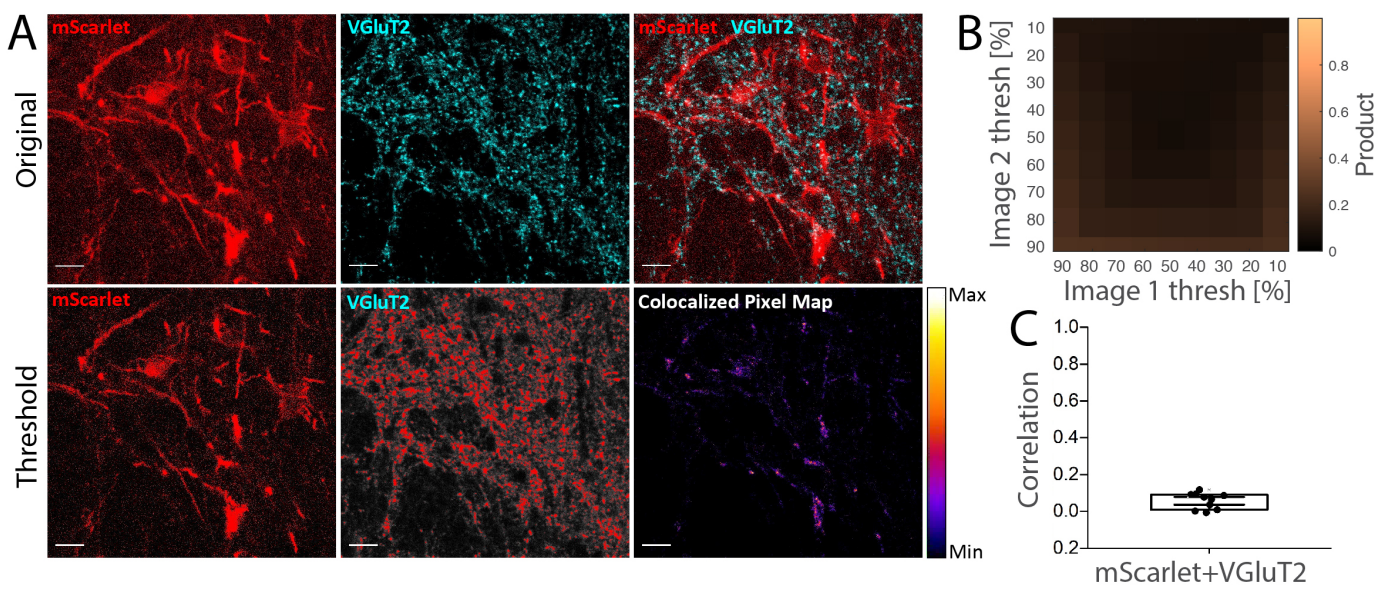

Fig. 5. CamKIIa has little or no colocalization with VGluT2 staining. (A) Top row: The infected cells with the CamKIIa promoter express $\mathrm{mScarlet}$. The immunstaining of the VGlut2 (cyan, middle) has little overlap with mScarlet (right). Bottom row: The threshold graphs and the heat map for colocalization. (B) The correlation for various levels of threshold appear dark. (C) The colocalization quantified as the correlation is rather low $(\mathrm{N}=3, \mathrm{n}=3-4)$. Scale bar $=20 \mu \mathrm{m}$. Note that $\mathrm{N}$ indicates the number of spinal cords and $\mathrm{n}$ is the number of slices analysed per spinal cord.

were visualized under the fluorescent microscope.

\section{Western blot}

Four weeks after the viral injection, the rats were deeply anesthetized and euthanized by cervical dislocation. Then the tissue samples were promptly collected on ice and reserved at $-80^{\circ} \mathrm{C}$ until further usage. The samples were homogenized with $1 \mathrm{X}$ RIPA lysis buffer $(50 \mathrm{mM} \mathrm{NaCl}, 150 \mathrm{mM}$ Tris- $\mathrm{HCl}$ at $\mathrm{pH} 7.5,5 \mathrm{mM}$ EDTA, $0.5 \%$ sodium deoxycholate, $1 \% \mathrm{NP}-40,0.1 \%$ sodium docecyl-sulfate), stirred $\left(30 \mathrm{~min}, 4^{\circ} \mathrm{C}\right)$ and centrifuged at $20,000 \mathrm{~g}\left(20 \mathrm{~min}, 4{ }^{\circ} \mathrm{C}\right)$. Protein quantification was performed using the bicinchoninic acid (BCA) method. $30 \mu \mathrm{g}$ protein was loaded per well and the concentration was normalized using loading buffer (2xSDS buffer: 2M DTT, 5xSDS, MilliQ) to reach $25 \mu 1$ total volume. Samples were vortexed and boiled for 6 min. Samples were loaded and electrophoresed in a 12\% SDS-polyacrylamide gel (Bio-Rad Laboratories, CA, USA) and transferred to the membrane (PVDF, Immobilon-P, Millipore, Billerica, MA, USA). The membranes were blocked 5\% milk in Tris-buffered saline Tween-20 (TBST), $1 \mathrm{~h}$ at room temperature. The following primary antibodies were applied for overnight at $4^{\circ} \mathrm{C}$ : rabbit anti-beta actin (1:100000, A3854 Sigma) and chicken anti-GFP (1:5000, AB13970, Abcam). Secondary antibodies were applied the next day: HRP donkey conjugated-anti-rabbit (1:10000, 31430 Thermo Scientific) and $\operatorname{IgY}(\mathrm{H}+\mathrm{L})$ goat anti-chicken cross-adsorbed antibody, (1:500, Alexa Fluor Plus 488 Thermo Scientific). Finally, membranes were washed 5 times in the washing buffer with TBST every $5 \mathrm{~min}$ and developed. The target proteins were detected by chemiluminescence (ECL).

\section{Immunohistochemistry}

After a month of virus injection, rats were deeply anesthetized using pentobarbital administered intraperitoneally (i.p) and transcardial perfusion was performed using 1X PBS followed by 4\% paraformaldehyde (PFA). The fixed spinal cords were removed out and further kept in PFA for $4 \mathrm{~h}$ followed by cryoprotected in 30\% (w/v) sucrose for later use (El Waly et al, 2021). $20 \mu \mathrm{m}$ transverse lumbar spinal slices were cut using cryostat, collected on Superfrost plus glass slides (Thermo Fisher Scientific GmbH, Germany) and stored at $-20{ }^{\circ} \mathrm{C}$. The whole procedure was performed under moist conditions in dark and was obtained from prior reports ${ }^{27,28}$. The spinal slices were washed, incubated with blocking solution (5\% fetal bovine serum, 5\% bovine serum albumin, $1 \%$ PBS, $0.3 \%$ Triton X-100) at room temperature for 1 to $2 \mathrm{~h}$ and primary antibodies were applied for overnight $\left(4^{\circ} \mathrm{C}\right)$. The following antibodies were used such as NeuN (rabbit monoclonal; 1:500 dilution; abcam AB177487), anti-GFP (chicken polyclonal, 1:1000 dilution; abcam AB13970), GAD65 (rabbit polyclonal; 1:500 dilution; Sigma G5038), GAD67 (mouse monoclonal; 1:1000 dilution; abcam AB26116), Pax2 (rabbit polyclonal; 1:500; Invitrogen UD283859), SMI32 (mouse monoclonal; 1:1000 dilution; EMD Millipore; 3256992), ChAT (goat polyclonal; 1:500; EMD Millipore AB144P), VGluT1 (mouse, 1:1000 dilution, synaptic Systems, GmbH, 407 Goettingen, Germany, 135011), VGluT2 (rabbit monoclonal; 1:500 dilution; abcam ab216463), GluA1 clone C3T (rabbit monoclonal, 1:500; EMD Millipore 04-855), GluA2 (mouse monoclonal; 1:200; EMD Millipore MAB397). Subsequent day, the slices were washed and incubated with secondary antibodies (1:500 dilution), donkey anti- rabbit Alexa Fluor 594 (Invitrogen R37119), donkey anti-chicken Alexa Fluor 488 

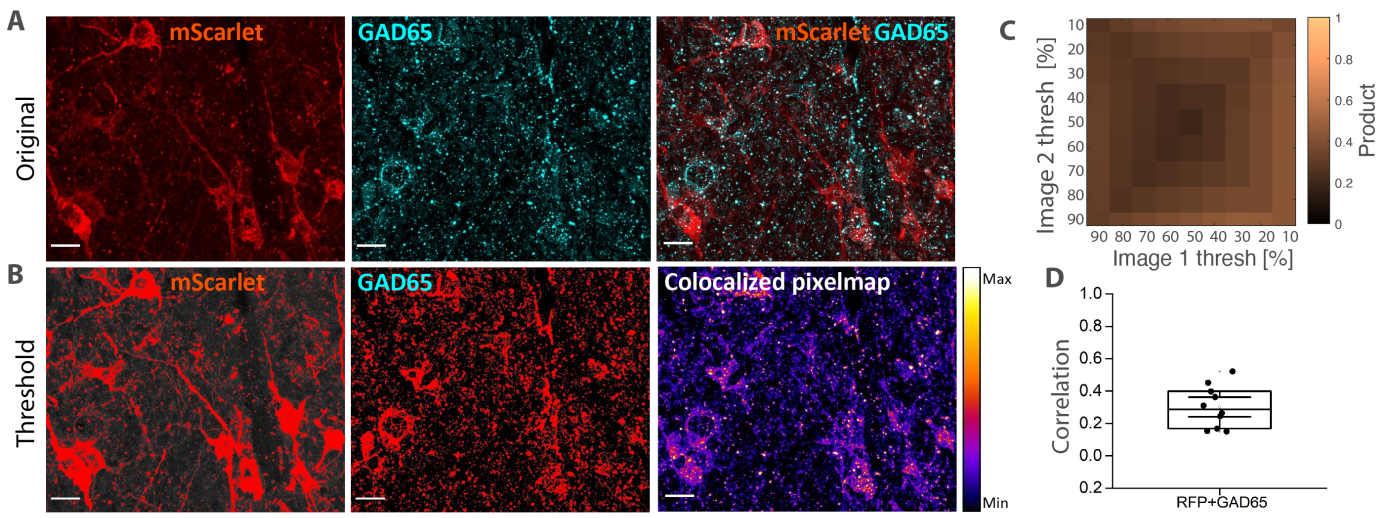

Fig. 6. CamKIIa-infected cells has poor overlap with GAD65 expression. (A) Top row: mScarlet expression (red, CamKIIa promotor) and the immunostaining of GAD65 (cyan) has only little colocalization. (B) Quantification of colocalization using the threshold of the fluorescent signal. (C) The mosaic of correlation coefficients for various thresholds, indicate poor overlap. (D) Sample correlation is weak $(N=3, n=3-4)$. Scale bar $=20 \mu \mathrm{m}$. Note that $\mathrm{N}$ is the number of spinal cords and $\mathrm{n}$ indicates the number of slices analysed per spinal cord.

(Jackson ImmunoResearch 703-546-155), donkey anti-mouse Alexa Fluor 647 (Invitrogen, A31571), donkey anti goat (abcam AB150132) and DAPI (1:1000 dilution applied for $2 \mathrm{hr}$; Sigma-Aldrich) at room temperature for 1 to $2 \mathrm{~h}$. Mounting was performed using DAKO mounting medium and the slices were visualized using Axio scan.Z1 and confocal microscopes using 20X magnification. Captured images were finally processed using Zen Lite 3.1 and ImageJ softwares.

\section{Quantification and statistics}

Quantification to verify the AAV viral co-expression with neuronal biomarkers was performed by manually counting the neurons in the $\mathrm{D}, \mathrm{C}$ and $\mathrm{V}$ spinal cord followed by computing the percentage of co-localization. The co-expression was further computed by using the colocalization threshold and Ezcolocolization plugins in Image ${ }^{17}$. Pearson correlation coefficient was computed ${ }^{29-31}$. Graphs were made using commercially available software (Origin2020, Origin Lab) and the plotted values are shown as percent change of a new value with respect to the original value.

\section{Acknowledgements}

We would like to thank Core Facility for Integrated Microscopy (CFIM) for providing us imaging facility and Søren Heide Jørgensen for making the virus. We would also like to thank Amaia de Diego Ajenjo from Dr. David Paul Drucker Woldbye Lab from Neuroscience department at the University of Copenhagen for providing us some mice brains with mDlx expression.

\section{Conflict of interest}

The authors declare no conflicts of interest. 

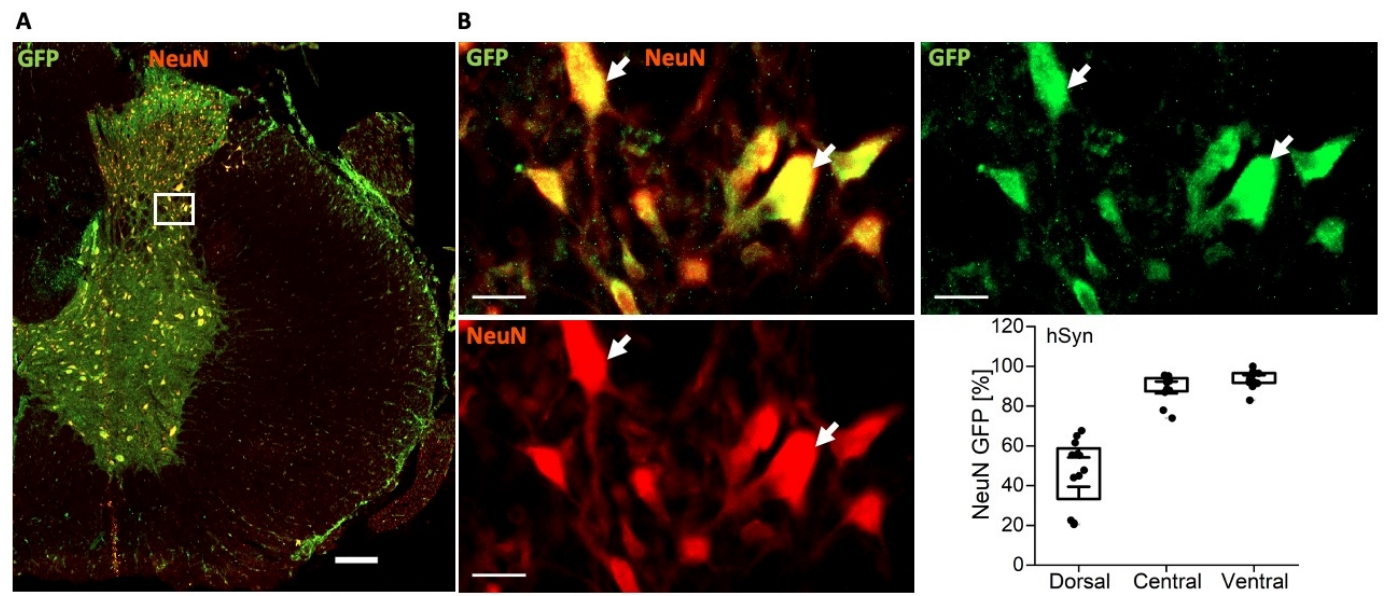

Fig. 7. hSyn1 targets a large fraction of spinal neurons. (A) A spinal section with infection using hSyn1-promotor co-stained with NeuN marker for neurons. (B) Highlighted region in (A) shows the extensive overlap between the infected cells and the NeuN marker. Scale bar $=200 \mu \mathrm{m}$ in image A and $20 \mu \mathrm{m}$ in the zoom-in images (B). $\mathrm{N}=4, \mathrm{n}=3$ where $\mathrm{N}$ indicates the number of spinal cords used and $\mathrm{n}$ is the number of slices analysed per spinal cord.

\section{References}

1. Luo, L., Callaway, E. M. \& Svoboda, K. Genetic Dissection of Neural Circuits: A Decade of Progress. Neuron 98, 256-281, DOI: 10.1016/j.neuron.2018.03.040 (2018).

2. Kügler, S., Kilic, E. \& Bähr, M. Human synapsin 1 gene promoter confers highly neuron-specific long-term transgene expression from an adenoviral vector in the adult rat brain depending on the transduced area. Gene Ther. 10, 337-347, DOI: 10.1038/sj.gt.3301905 (2003).

3. Pla, R. et al. Dlx1 and Dlx2 Promote Interneuron GABA Synthesis, Synaptogenesis, and Dendritogenesis. Cereb. Cortex 28, 3797-3815, DOI: 10.1093/cercor/bhx241 (2018).

4. Panganiban, G. \& Rubenstein, J. L. Developmental functions of the Distal-less/Dlx homeobox genes. Development 129, 4371-4386 (2002).

5. Le, T. N. et al. Dlx homeobox genes promote cortical interneuron migration from the basal forebrain by direct repression of the semaphorin receptor neuropilin-2. J. Biol. Chem. DOI: 10.1074/jbc.M607486200 (2007).

6. Wilson, D. E. et al. GABAergic Neurons in Ferret Visual Cortex Participate in Functionally Specific Networks. Neuron 93, 1058-1065, DOI: 10.1016/j.neuron.2017.02.035 (2017).

7. Dimidschstein, J. et al. A viral strategy for targeting and manipulating interneurons across vertebrate species. Nat. Neurosci. 19, 1743-49, DOI: 10.1038/nn.4430 (2016).

8. Dobrott, C. I., Sathyamurthy, A. \& Levine, A. J. Decoding cell type diversity within the spinal cord. Curr. Opin. Physiol. 8, 1-6, DOI: 10.1016/j.cophys.2018.11.006 (2019).

9. Russ, D. E. et al. A harmonized atlas of mouse spinal cord cell types and their spatial organization. Nat. Commun. 12, DOI: $10.1038 / \mathrm{s} 41467-021-25125-1$ (2021).

10. Berg, R. W., Willumsen, A. \& Lindén, H. When networks walk a fine line: balance of excitation and inhibition in spinal motor circuits. Curr. Opin. Physiol. 8, 76-83, DOI: 10.1016/j.cophys.2019.01.006 (2019).

11. Mazzone, G. L., Mohammadshirazi, A., Aquino, J. B., Nistri, A. \& Taccola, G. GABAergic Mechanisms Can Redress the Tilted Balance between Excitation and Inhibition in Damaged Spinal Networks. Mol. Neurobiol. 58, 3769-3786, DOI: 10.1007/s12035-021-02370-5 (2021).

12. Lindén, H. \& Berg, R. W. Why Firing Rate Distributions Are Important for Understanding Spinal Central Pattern Generators. Front. Hum. Neurosci. 15, 719388, DOI: 10.3389/fnhum.2021.719388 (2021). 

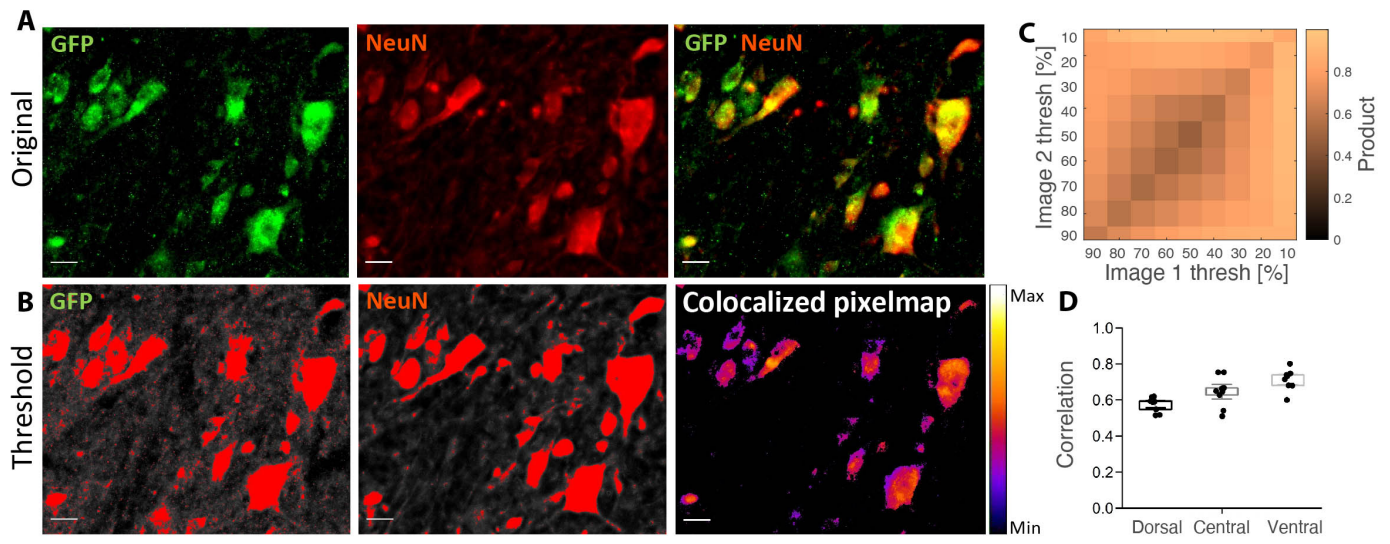

Fig. 8. hSyn1-driven infection colocalization with NeuN biomarker. (A) Top row: Expression of GFP with hSyn1 promotor has high overlap with the NeuN staining. (B) Threshold and colocalization in a heat map. (C-D) The colocalization is quantified for different thresholds and across the three regions. Scale bar $=20 \mu \mathrm{m} . \mathrm{N}=3, \mathrm{n}=3$, where $\mathrm{N}$ is the number of spinal cords and $\mathrm{n}$ is the number of slices analysed per spinal cord.

13. Aboitiz, F. \& Montiel, J. Origin of the Mammalian Brain. In: Origin and Evolution of the Vertebrate Telencephalon, with Special Reference to the Mammalian Neocortex. Adv. anatomy, embryology, cell biology 193, DOI: 10.1007/ 978-3-540-49761-5 (2007).

14. Chettih, S. N. \& Harvey, C. D. Single-neuron perturbations reveal feature-specific competition in V1. Nature 567, 334-340, DOI: 10.1038/s41586-019-0997-6 (2019).

15. Shemesh, O. A. et al. Publisher Correction: Temporally precise single-cell-resolution optogenetics (Nature Neuroscience (2017) DOI: 10.1038/s41593-017-0018-8). Nat. Neurosci. 21, 896, DOI: 10.1038/s41593-018-0097-1 (2018).

16. Burrill, J. D., Moran, L., Goulding, M. D. \& Saueressig, H. PAX2 is expressed in multiple spinal cord interneurons, including a population of EN1+ interneurons that require PAX6 for their development. Development 124, 4493-4503, DOI: $10.1242 /$ dev.124.22.4493 (1997).

17. Stauffer, W., Sheng, H. \& Lim, H. N. EzColocalization: An ImageJ plugin for visualizing and measuring colocalization in cells and organisms. Sci. Reports 8, DOI: 10.1038/s41598-018-33592-8 (2018).

18. Todd, A. J. et al. The expression of vesicular glutamate transporters VGLUT1 and VGLUT2 in neurochemically defined axonal populations in the rat spinal cord with emphasis on the dorsal horn. Eur. J. Neurosci. 17, 13-27, DOI: 10.1046/j.1460-9568.2003.02406.x (2003).

19. Bikoff, J. B. et al. Spinal Inhibitory Interneuron Diversity Delineates Variant Motor Microcircuits. Cell 165, 207-219, DOI: 10.1016/j.cell.2016.01.027 (2016).

20. Gabitto, M. I. et al. Bayesian Sparse Regression Analysis Documents the Diversity of Spinal Inhibitory Interneurons. Cell 165, 220-233, DOI: 10.1016/j.cell.2016.01.026 (2016).

21. Mackie, M., Hughes, D. I., Maxwell, D. J., Tillakaratne, N. J. \& Todd, A. J. Distribution and colocalisation of glutamate decarboxylase isoforms in the rat spinal cord. Neuroscience 119, 461-472, DOI: 10.1016/S0306-4522(03)00174-X (2003).

22. Lee, H. J. et al. Comparison of GAD65 and 67 Immunoreactivity in the Lumbar Spinal Cord Between Young Adult and Aged Dogs. Neurochem. Res. 36, DOI: 10.1007/s11064-010-0361-6 (2011).

23. Deuchars, S. A. GABAergic Neurons in the Central Region of the Spinal Cord: A Novel Substrate for Sympathetic Inhibition. J. Neurosci. 25, DOI: 10.1523/JNEUROSCI.3740-04.2005 (2005).

24. Zhang, S. \& Morales, M. Identification of glutamatergic neurons. Neuromethods 130, 1-28, DOI: 10.1007/ 978-1-4939-7228-9\{_\}1 (2018).

25. Chaterji, S., Barik, A. \& Sathyamurthy, A. Intraspinal injection of adeno-associated viruses into the adult mouse spinal cord. STAR Protoc. 2, DOI: 10.1016/j.xpro.2021.100786 (2021).

26. Haenraets, K., Albisetti, G. W., Foster, E. \& Wildner, H. Adeno-associated virus-mediated transgene expression in genetically defined neurons of the spinal cord. J. Vis. Exp. 135, 1-11, DOI: 10.3791/57382 (2018). 
27. Kaur, J., Flores Gutiérrez, J. \& Nistri, A. Neuroprotective effect of propofol against excitotoxic injury to locomotor networks of the rat spinal cord <i>in vitro</i>. Eur. J. Neurosci. 44, DOI: 10.1111/ejn.13353 (2016).

28. Kaur, J., Rauti, R. \& Nistri, A. Nicotine-mediated neuroprotection of rat spinal networks against excitotoxicity. Eur. J. Neurosci. 47, DOI: 10.1111/ejn.13950 (2018).

29. Kaur, J., Mazzone, G. L., Aquino, J. B. \& Nistri, A. Nicotine Neurotoxicity Involves Low Wnt1 Signaling in Spinal Locomotor Networks of the Postnatal Rodent Spinal Cord. Int. J. Mol. Sci. 22, DOI: 10.3390/ijms22179572 (2021).

30. Costes, S. V. et al. Automatic and Quantitative Measurement of Protein-Protein Colocalization in Live Cells. Biophys. J. 86, DOI: 10.1529/biophysj.103.038422 (2004).

31. MANDERS, E. M. M., VERBEEK, F. J. \& ATEN, J. A. Measurement of co-localization of objects in dual-colour confocal images. J. Microsc. 169, DOI: 10.1111/j.1365-2818.1993.tb03313.x (1993). 


\section{Supplementary Files}

This is a list of supplementary files associated with this preprint. Click to download.

- ViraltargetingGABAergicneuronssupplementarymaterial.pdf 